\title{
Partially polarized pseudo-Schell model sources
}

Rosario Martínez-Herrero, David Maluenda, Gemma Piquero, Juan Carlos G. de Sande, Massimo Santarsiero, et al. 


\title{
Partially polarized pseudo-Schell model sources
}

\author{
Rosario Martínez-Herrero ${ }^{a}$, David Maluenda ${ }^{a}$, Gemma Piquero $^{a}$, Juan Carlos G. de Sande ${ }^{b}$, \\ Massimo Santarsiero ${ }^{c}$ and Franco Gori ${ }^{c}$ \\ ${ }^{a}$ Departamento de Óptica, Universidad Complutense de Madrid, Ciudad Universitaria, 28040 \\ Madrid, Spain; \\ ${ }^{b}$ ETSIS de Telecomunicación, Universidad Politécnica de Madrid, Campus Sur 28031 Madrid, \\ Spain; \\ ${ }^{c}$ Dipartimento di Ingegneria, Università Roma Tre, Via V. Volterra 62, 00146 Rome, Italy
}

\begin{abstract}
In this work a new type of partially polarized and partially coherent sources is proposed. The coherence characteristics of these sources are dependent on the difference of the radial distances from the source center of the two points to be compared. The coherence is perfect for points located on the same circle centered on the source center and decreases for points that belongs to different concentric circles. The maximum attainable coherence is related to the degree of polarization of the source. Coherence and polarization characteristics of this kind of fields at the source plane and upon free space propagation are analyzed in detail for a simple case. For the particular presented example, a partially polarized and partially coherent field is obtained, whose polarization properties are invariant in propagation.
\end{abstract}

Keywords: Coherence, Polarization, Beam propagation

\section{INTRODUCTION}

There is an increasing interest in finding physically realizable partially coherent sources with coherence characteristics different from those of the usual Schell model type. ${ }^{1-14}$ It is also expected that interesting results could be found if the vectorial nature of light is taken into account. The coherence and polarization characteristics of a light source can be appropriately described by its cross-spectral density matrix (CSDM). ${ }^{15,16}$ Recent findings of the coherency theory, such as the superposition rule and its generalization to the vectorial case, ${ }^{1-3,17}$ have eased the study of new realizable CSDM's.

In this work a new type of sources is proposed, which are both partially polarized and partially coherent. Their coherence characteristics are not shift invariant, as it occurs for sources of the Schell-model type, but depend on the difference between the moduli of the two point vectors considered. ${ }^{13}$ We shall call them polarized pseudo-Schell model sources. The coherence and polarization characteristics of this kind of fields at the plane source and upon free space propagation will be analyzed in detail.

\section{KEY DEFINITIONS}

The cross-spectral density matrix $\widehat{G}\left(\mathbf{r}_{\mathbf{1}}, \mathbf{r}_{\mathbf{2}}\right)$ is an approprite tool for describing stochastic, statistically stationary up to second order, electromagnetic light sources. ${ }^{15,16}$ Under paraxial approximation, the CSDM is a $2 \times 2$ matrix that gives the correlations between different field components at two arbitrary positions across the source plane. The two points are denoted by the vectors $\mathbf{r}_{j}=\left(r_{j}, \theta_{j}\right)$ with $j=1,2$ where $\left(r_{j}, \theta_{j}\right)$ are the radial and angular coordinates, respectively.

Further author information: (Send correspondence to D.M.)

D. M.: E-mail: d.maluenda@ucm.es

R.M.H.: E-mail: r.m-h@fis.ucm.es

G.P.: E-mail: piquero@ucm.es

J.C.G.S.: E-mail: jcgsande@ics.upm.es

M.S.: E-mail: massimo.santarsiero@uniroma3.it

M.S.: E-mail: franco.gori@uniroma3.it

Third International Conference on Applications of Optics and Photonics, edited by Manuel F. M. Costa, Proc. of SPIE Vol. 10453, 104531N · C 2017 SPIE · CCC code: 0277-786X/17/\$18 · doi: 10.1117/12.2272154 
In the scalar treatment, a genuine cross-spectral density must satisfy the nonnegativeness condition. ${ }^{15}$ However, to check directly this nonnegativeness condition for a given cross-spectral density is not an easy task. Some years ago a necessary and sufficient condition that guarantees such nonnegativeness has been established. ${ }^{3,17}$ This condition gives a rule, the so-called superposition rule, ${ }^{17}$ to easily construct physically realizable crossspectral densities. An extension of this rule for the electromagnetic case has been derived, ${ }^{1,2}$ so it can be easily checked if a proposed matrix represents a genuine CSDM.

The coherence properties of an electromagnetic source can be studied through the electromagnetic degree of coherence $^{18-20}$

$$
\mu_{S T F}^{2}\left(\mathbf{r}_{1}, \mathbf{r}_{2}, z\right)=\frac{\operatorname{tr}\left\{\widehat{G}\left(\mathbf{r}_{1}, \mathbf{r}_{2}, z\right) \widehat{G}^{\dagger}\left(\mathbf{r}_{1}, \mathbf{r}_{2}, z\right)\right\}}{\operatorname{tr}\left\{\widehat{G}\left(\mathbf{r}_{1}, \mathbf{r}_{1}, z\right)\right\} \operatorname{tr}\left\{\widehat{G}\left(\mathbf{r}_{2}, \mathbf{r}_{2}, z\right)\right\}},
$$

where "tr" and $\dagger$ denote the trace and the transpose conjugate of a matrix, respectively.

The evaluation of the CSDM at the same point $\mathbf{r}=\mathbf{r}_{\mathbf{1}}=\mathbf{r}_{\mathbf{2}}$ results in the polarization matrix ${ }^{15}$ that describes all the polarization characteristics of the field at any point in its cross section. On the other hand, the polarization properties of light beams are usually studied through a set of four parameters usually arranged in a vector, the so-called Stokes vector $\mathbf{S}=\left(S_{0}, S_{1}, S_{2}, S_{3}\right)^{T},{ }^{21,22}$ where superscript $T$ denotes transpose. In general, $\mathbf{S}$ is a point-dependent vector that can be derived from the polarization matrix as ${ }^{15}$

$$
S_{i}(\mathbf{r}, z)=\operatorname{tr}\left\{\widehat{G}(\mathbf{r}, \mathbf{r}, z) \widehat{\sigma}_{i}\right\}
$$

where $\widehat{\sigma}_{i},(i=0,1,2,3)$, are the $2 \times 2$ identity matrix together with the three Pauli matrices ${ }^{15}$

$$
\widehat{\sigma}_{0}=\left(\begin{array}{cc}
1 & 0 \\
0 & 1
\end{array}\right) ; \quad \widehat{\sigma}_{1}=\left(\begin{array}{cc}
1 & 0 \\
0 & -1
\end{array}\right) ; \widehat{\sigma}_{2}=\left(\begin{array}{cc}
0 & 1 \\
1 & 0
\end{array}\right) ; \widehat{\sigma}_{3}=\left(\begin{array}{cc}
0 & -\mathrm{i} \\
\mathrm{i} & 0
\end{array}\right) \text {. }
$$

The Stokes vector gives the same information as that contained in the polarization matrix, but the components of the former are measurable quantities. For example, $S_{0}(\mathbf{r}, z)=\operatorname{tr}\{\widehat{G}(\mathbf{r}, \mathbf{r}, z)\}$ is the source intensity, and the remaining three represent the source intensity measured with suitable wave plates and linear polarizers placed in front of the detector.

The degree of polarization, $P$, which is the ratio of the polarized part of the field to the total irradiance, ${ }^{15}$ gives important information about the polarization of the field in a single parameter. It can be obtained at each point of the cross section from the polarization matrix as

$$
P(\mathbf{r}, z)=\sqrt{1-\frac{4 \operatorname{det}\{\widehat{G}(\mathbf{r}, \mathbf{r}, z)\}}{(\operatorname{tr}\{\widehat{G}(\mathbf{r}, \mathbf{r}, z)\})^{2}}},
$$

where "det" stands for determinant.

To study the polarization and coherence characteristics of the field upon free space propagation, we assume paraxial approximation and propagation direction mainly along the $z$ axis of a suitable reference frame. Then, the Fresnel diffraction integral ${ }^{15}$ allows us to obtain the CSDM at any plane $z=$ const. From the knowledge of the CSDM at the source plane $(z=0)$, it turns out that ${ }^{15}$

$$
\widehat{G}\left(\mathbf{R}_{1}, \mathbf{R}_{2}, z\right)=\frac{1}{\lambda^{2} z^{2}} \iint \widehat{G}\left(\mathbf{r}_{1}, \mathbf{r}_{2}, 0\right) \exp \left[-\frac{\mathrm{i}}{2 z}\left(\left|\mathbf{R}_{1}-\mathbf{r}_{1}\right|^{2}-\left|\mathbf{R}_{2}-\mathbf{r}_{2}\right|^{2}\right)\right] d^{2} r_{1} d^{2} r_{2},
$$

where $\mathbf{R}_{j}=\left(R_{j}, \phi_{j}\right)$, with $j=1,2$, are two arbitrary position vectors across the plane $z=$ const. 


\section{SOURCE MODEL}

Let us consider a source at the plane $z=0$ described by a matrix of the form

$$
\widehat{G}\left(\mathbf{r}_{1}, \mathbf{r}_{2}, 0\right)=\tau\left(\mathbf{r}_{1}\right)^{*} \tau\left(\mathbf{r}_{2}\right) \int_{0}^{\infty} \int_{0}^{2 \pi} \widehat{A}^{\dagger}\left(\mathbf{r}_{1}, \boldsymbol{\sigma}\right) \widehat{A}\left(\mathbf{r}_{2}, \boldsymbol{\sigma}\right) \eta d \eta d \alpha,
$$

where $\mathbf{r}_{j}=\left(r_{j}, \theta_{j}\right)$ with $j=1,2$ are two arbitrary positions across the source plane, $\boldsymbol{\sigma}=(\eta, \alpha), \tau(\mathbf{r})$ is a complex function, and

$$
\widehat{A}\left(\mathbf{r}_{1}, \boldsymbol{\sigma}\right)=\widehat{H}(\eta) \exp (\mathrm{i} k r \eta \cos \alpha),
$$

being $\widehat{H}(\eta)$ an arbitrary $2 \times 2$ matrix, i the imaginary unit, and $k$ the wave number. Due to the way in which $\widehat{G}\left(\mathbf{r}_{1}, \mathbf{r}_{2}\right)$ has been constructed, it represents a genuine cross spectral density matrix. ${ }^{1-3}$

Substitution from Eq. (7) into Eq. (6) gives

$$
\widehat{G}\left(\mathbf{r}_{1}, \mathbf{r}_{2}, 0\right)=\tau\left(\mathbf{r}_{1}\right)^{*} \tau\left(\mathbf{r}_{2}\right) \widehat{G}_{0}\left(r_{2}-r_{1}\right),
$$

where

$$
\widehat{G}_{0}\left(r_{2}-r_{1}\right)=2 \pi \int_{0}^{\infty} \widehat{H}^{\dagger}(\eta) \widehat{H}(\eta) J_{0}\left[k \eta\left(r_{2}-r_{1}\right)\right] \eta d \eta,
$$

and $J_{\nu}(\cdot)$ is the Bessel function of first kind and order $\nu{ }^{23}$ It can be noted that the intensity of the source, except for a constant factor, is determined by the function $\tau(\mathbf{r})$, while its polarization and coherence properties are determined by the matrix $\widehat{G}_{0}\left(r_{2}-r_{1}\right)$.

To illustrate the properties of this kind of sources, we consider the following particular choice for $\widehat{H}(\eta)$ :

$$
\widehat{H}(\eta)=\left(\begin{array}{cc}
h_{x x} & 0 \\
0 & h_{y y}
\end{array}\right) \operatorname{circ}\left(\frac{\eta}{b}\right)
$$

being $h_{x x}$ and $h_{x x}$ two arbitrary dimensionless constant coefficients, $\operatorname{circ}(t)=1$ for $|t| \leq 1$ and 0 otherwise, and $b$ a parameter related to the area where the coherence of the source is significantly high.

For this choice of $\widehat{H}(\eta)$ we obtain

$$
\widehat{G}_{0}\left(r_{2}-r_{1}\right)=\left(\begin{array}{cc}
\left|h_{x x}\right|^{2} & 0 \\
0 & \left|h_{y y}\right|^{2}
\end{array}\right) \pi b^{2} \frac{2 J_{1}\left[b k\left(r_{2}-r_{1}\right)\right]}{b k\left(r_{2}-r_{1}\right)},
$$

and the squared electromagnetic degree of coherence across the source turns out to be

$$
\mu_{S T F}^{2}(z=0)=\frac{\left|h_{x x}\right|^{4}+\left|h_{y y}\right|^{4}}{\left(\left|h_{x x}\right|^{2}+\left|h_{y y}\right|^{2}\right)^{2}}\left[\frac{2 J_{1}\left[b k\left(r_{2}-r_{1}\right)\right]}{b k\left(r_{2}-r_{1}\right)}\right]^{2} .
$$

It reaches its maximum value, $\mu_{S T F, \operatorname{Max}}^{2}(z=0)$, equal to

$$
\mu_{S T F, M a x}^{2}(z=0)=\frac{\left|h_{x x}\right|^{4}+\left|h_{y y}\right|^{4}}{\left(\left|h_{x x}\right|^{2}+\left|h_{y y}\right|^{2}\right)^{2}},
$$

when $r_{2}=r_{1}$, that is, for concentric rings around the source center. From this maximum value, the electromagnetic degree of coherence decreases and presents an oscillatory behavior for increasing values of $\left|r_{2}-r_{1}\right|$. The distance $\left|r_{2}-r_{1}\right|$ for which the first null of the electromagnetic degree of coherence is reached depends on the single parameter $b$.

On the other hand, the polarization properties are determined by the following Stokes vector

$$
\mathbf{S}(\mathbf{r}, z=0)=|\tau(\mathbf{r})|^{2} \pi b^{2}\left(\begin{array}{c}
\left|h_{x x}\right|^{2}+\left|h_{y y}\right|^{2} \\
\left|h_{x x}\right|^{2}-\left|h_{y y}\right|^{2} \\
0 \\
0
\end{array}\right)
$$


that results in a uniformly partially polarized field with degree of polarization given by

$$
P(z=0)=\frac{\left.|| h_{x x}\right|^{2}-\left|h_{y y}\right|^{2} \mid}{\left|h_{x x}\right|^{2}+\left|h_{y y}\right|^{2}}
$$

It can be noted that the degree of polarization can vary from zero, for $\left|h_{x x}\right|^{2}=\left|h_{y y}\right|^{2}$, to one, when $\left|h_{y y}\right|^{2}=0$ or $\left|h_{x x}\right|^{2}=0$. Then, it is possible to generate unpolarized or totally polarized fields by varying the values of $h_{x x}$ and $h_{y y}$. For different values of the coefficients $h_{x x}$ and $h_{y y}$, the field is partially polarized.

Comparing Eqs. (13) and (15), it can be noted that the maximum attainable electromagnetic degree of coherence is related to the degree of polarization of the source in the following way

$$
\mu_{S T F, M a x}^{2}(z=0)=\frac{1+P^{2}(z=0)}{2} .
$$

This means that, for totally polarized sources of this kind, the field is completely coherent $\left(\mu_{S T F}^{2}=1\right)$ for all points on a ring concentric to the source center $r_{2}=r_{1}$. For the case of partially polarized sources belonging to the proposed class, the field is partially coherent even for $r_{2}=r_{1}$. For the limiting case of unpolarized source, the maximum attainable electromagnetic degree of coherence is $1 / 2$.

\section{FREE SPACE PROPAGATION}

For the sources described in the previous section, the properties of field generated under free space propagation can be obtained assuming paraxial approximation. If we consider a source with $\tau(\mathbf{r})=\tau(r) \exp (\mathrm{i} m \theta)$, the CSDM at a given $z$ plane can be obtained by substituting Eq. (11) into Eq. (8) and the latter into Eq. (5). This gives

$$
\begin{aligned}
& \widehat{G}\left(\mathbf{R}_{1}, \mathbf{R}_{2}, z\right)=\frac{4 \pi^{2}}{\lambda^{2} z^{2}} \exp \left[\frac{\mathrm{i} k}{2 z}\left(R_{2}^{2}-R_{1}^{2}\right)\right] \exp \left[\mathrm{i} m\left(\phi_{2}-\phi_{1}\right)\right]\left(\begin{array}{cc}
\left|h_{x x}\right|^{2} & 0 \\
0 & \left|h_{y y}\right|^{2}
\end{array}\right) \frac{b^{2}}{2} \\
& \quad \times \int_{0}^{\infty} \int_{0}^{\infty} \tau\left(r_{1}\right)^{*} \tau\left(r_{2}\right) \exp \left[\frac{\mathrm{i} k}{2 z}\left(r_{2}{ }^{2}-r_{1}{ }^{2}\right)\right] \frac{2 J_{1}\left(k b\left(r_{2}-r_{1}\right)\right)}{k b\left(r_{2}-r_{1}\right)} J_{m}\left(\frac{k}{z} R_{1} r_{1}\right) J_{m}\left(\frac{k}{z} R_{2} r_{2}\right) r_{1} r_{2} d r_{1} d r_{2} .
\end{aligned}
$$

In general, the coherence characteristics of the field affect the polarization, and the degree of polarization usually changes during propagation. ${ }^{24-28}$ We propose a class of partially coherent field for which the polarization properties remains invariant upon free-space propagation. On the other hand, the intensity profile and the electromagnetic degree of coherence of these sources vary with propagation.

\section{SUMMARY}

In the present work, a new type of electromagnetic sources has been proposed. They are partially polarized and partially coherent. For the simple example developed in this work, the polarization properties of the source remain invariant in propagation. The degree of polarization modulates the electromagnetic degree of coherence, which presents an annular shape across the source plane: it reaches its maximum value for points at equal distance from the center of the source and varies in the form given in Eq. (12) for other points.

\section{ACKNOWLEDGMENTS}

Spanish Ministerio de Economía y Competitividad project FIS2016-75147. 


\section{REFERENCES}

[1] Gori, F., Santarsiero, M., Borghi, R., and Ramírez-Sánchez, V., "Realizability condition for electromagnetic Schell-model sources," J. Opt. Soc. Am. A 25, 1016-1021 (May 2008).

[2] Martínez-Herrero, R. and Mejías, P. M., "Elementary-field expansions of genuine cross-spectral density matrices," Opt. Lett. 34, 2303-2305 (Aug 2009).

[3] Martínez-Herrero, R., Mejías, P. M., and Gori, F., "Genuine cross-spectral densities and pseudo-modal expansions," Opt. Lett. 34, 1399-1401 (May 2009).

[4] Santarsiero, M., Ramírez-Sánchez, V., and Borghi, R., "Partially correlated thin annular sources: the vectorial case," J. Opt. Soc. Am. A 27, 1450- 1456 (Jun 2010).

[5] Pires, H. D. L., Woudenberg, J., and van Exter, M. P., "Measurements of spatial coherence of partially coherent light with and without orbital angular momentum," J. Opt. Soc. Am. A 27, 2630-2637 (Dec 2010).

[6] Lajunen, H. and Saastamoinen, T., "Propagation characteristics of partially coherent beams with spatially varying correlations," Opt. Lett. 36, 4104-4106 (Oct 2011).

[7] Cai, Y., Chen, Y., and Wang, F., "Generation and propagation of partially coherent beams with nonconventional correlation functions: a review [invited]," J. Opt. Soc. Am. A 31, 2083-2096 (Sep 2014).

[8] Santarsiero, M., Piquero, G., de Sande, J. C. G., and Gori, F., "Difference of cross-spectral densities," Opt. Lett. 39, 1713-1716 (Apr 2014).

[9] Borghi, R., Gori, F., Guattari, G., and Santarsiero, M., "Twisted Schell-model beams with axial symmetry," Opt. Lett. 40, 4504-4507 (Oct 2015).

[10] Chen, Y., Wang, F., Liu, L., Zhao, C., Cai, Y., and Korotkova, O., "Generation and propagation of a partially coherent vector beam with special correlation functions," Phys. Rev. A 89, 013801 (Jan 2014).

[11] Singh, M., Lajunen, H., Tervo, J., and Turunen, J., "Imaging with partially coherent light: elementary-field approach," Opt. Express 23, 28132-28140 (Nov 2015).

[12] de Sande, J. C. G., Santarsiero, M., Piquero, G., and Gori, F., "The subtraction of mutually displaced Gaussian Schell-model beams," Journal of Optics 17(12), 125613 (2015).

[13] Martínez-Herrero, R., Maluenda, D., Piquero, G., and de Sande, J. C. G., "Vortex pseudo schell-model source: A proposal," in [2016 15th Workshop on Information Optics (WIO)], 1-3 (July 2016).

[14] Hyde IV, M. W., Bose-Pillai, S., Xiao, X., and Voelz, D. G., "A fast and efficient method for producing partially coherent sources," Journal of Optics 19(2), 025601 (2017).

[15] Mandel, L. and Wolf, E., [Optical Coherence and Quantum Optics], Cambridge University Press (1995).

[16] Martínez-Herrero, R., Mejías, P. M., and Piquero, G., [Characterization of Partially Polarized Light Fields], Springer Series in Optical Science, Springer (2009).

[17] Gori, F. and Santarsiero, M., "Devising genuine spatial correlation functions," Opt. Lett. 32, 3531-3533 (Dec 2007).

[18] Tervo, J., Setälä, T., and Friberg, A. T., "Degree of coherence for electromagnetic fields," Opt. Express 11, 1137-1143 (May 2003).

[19] Setälä, T., Tervo, J., and Friberg, A. T., "Complete electromagnetic coherence in the space-frequency domain," Opt. Lett. 29, 328-330 (Feb 2004).

[20] Martínez-Herrero, R. and Mejías, P. M., "Relation between degrees of coherence for electromagnetic fields," Opt. Lett. 32, 1504-1506 (Jun 2007).

[21] Born, M. and Wolf, E., [Principles of Optics], Cambridge University Press, sixth (corrected) ed. (1980).

[22] Goldstein, D. H., [Polarized Light], Marcel Dekker, Inc., second (revised and expanded) ed. (2003).

[23] Abramowitz, M. and Stegun, I., eds., [Handbook of mathematical functions], Dover Publications Inc (1972).

[24] James, D. F. V., "Change of polarization of light beams on propagation in free space," J. Opt. Soc. Am. A 11, 1641-1643 (May 1994).

[25] Gori, F., Santarsiero, M., Piquero, G., Borghi, R., Mondello, A., and Simon, R., "Partially polarized Gaussian Schell-model beams," Journal of Optics A: Pure and Applied Optics 3(1), 1 (2001).

[26] Piquero, G., Gori, F., Romanini, P., Santarsiero, M., Borghi, R., and Mondello, A., "Synthesis of partially polarized Gaussian Schell-model sources," Optics Communications 208(13), 9 - 16 (2002). 
[27] de Sande, J. C. G., Santarsiero, M., Piquero, G., and Gori, F., "Longitudinal polarization periodicity of unpolarized light passing through a double wedge depolarizer," Opt. Express 20, 27348-27360 (Dec 2012).

[28] Santarsiero, M., de Sande, J. C. G., Piquero, G., and Gori, F., "Coherencepolarization properties of fields radiated from transversely periodic electromagnetic sources," Journal of Optics 15(5), 055701 (2013). 